\title{
GLAD!
}

Revue sur le langage, le genre, les sexualités

05 | 2018

Raconter les sexualités depuis la marge

\section{Lucille Gallardo \& Laura Spica (avec des illustrations de Claire Favre-Taylaz). 2014. La main devant le soleil. Expériences militantes et homosexualités en Afrique francophone}

Fleur Beauvieux

\section{OpenEdition}

Journals

Édition électronique

URL : http://journals.openedition.org/glad/1280

ISSN : 2551-0819

Éditeur

Association GSL

\section{Référence électronique}

Fleur Beauvieux, «Lucille Gallardo \& Laura Spica (avec des illustrations de Claire Favre-Taylaz). 2014 La main devant le soleil. Expériences militantes et homosexualités en Afrique francophone », GLAD! [En ligne], 05 | 2018, mis en ligne le 15 décembre 2018, consulté le 17 décembre 2020. URL : http:// journals.openedition.org/glad/1280

Ce document a été généré automatiquement le 17 décembre 2020.

\section{cc) $(1) \ominus$}

La revue GLAD! est mise à disposition selon les termes de la Licence Creative Commons Attribution -

Pas d'Utilisation Commerciale - Pas de Modification 4.0 International. 


\title{
Lucille Gallardo \& Laura Spica (avec des illustrations de Claire Favre- Taylaz). 2014. La main devant le soleil. Expériences militantes et homosexualités en Afrique francophone
}

\author{
Fleur Beauvieux
}

\section{RÉFÉRENCE}

Lucille Gallardo \& Laura Spica (avec des illustrations de Claire Favre-Taylaz). 2014. La main devant le soleil. Expériences militantes et homosexualités en Afrique francophone.

Marseille : Les éditions du Tamis. 136 pages.

Parce que l'homosexualité n'est pas partout légale et reconnue, La main devant le soleil. Expériences militantes et homosexualités en Afrique francophone, paru en 2014, constitue un ouvrage clé pour tenter de comprendre comment les acteur.es existent et la vivent dans des contextes où les libertés individuelles sont encore trop souvent bafouées. Tiré d'une étude commanditée par AIDES et Sidaction et réalisée par Lucille Gallardo entre 2011 et 2012, ayant consisté en la réalisation de 122 entretiens semi-directifs dans sept pays d'Afrique francophone (Burkina Faso, Côte d'Ivoire, Togo, Algérie, Maroc, Cameroun et Burundi), ce livre propose une restitution originale d'une partie de ce travail. Puisqu'il est rare d'entendre la parole de celles et ceux qui s'engagent pour la cause homosexuelle autrement qu'en des extraits d'entretiens, La main devant le soleil nous offre sept récits - retravaillés par deux anthropologues (Lucille Gallardo et Laura Spica) - de militant.es qui luttent au quotidien, à partir de leur combat contre le virus $\mathrm{VIH} /$ sida. En découle un ouvrage qui allie rigueur méthodologique et esthétique de la restitution, puisque le livre est illustré à l'encre de Chine par Claire Favre-Taylaz. 
2 Après une brève introduction ( « Du déni à la reconnaissance ») rappelant le retard de la prise en compte des pratiques homosexuelles dans la propagation du virus en Afrique par rapport à d'autres parties du monde, et les différents enjeux autour de cette lente reconnaissance, l'ouvrage est structuré en trois parties ("Des normes à la pratique »; «Parcours d'émergence» et "Travailler auprès de ses pairs»). Les récits de vie constituent les trois quarts du livre. Des pages annexes exposent la méthodologie de l'étude de départ et la façon dont les entretiens ont été retravaillés. La main devant le soleil touche aux domaines de l'anthropologie de la santé, de l'anthropologie politique et de l'anthropologie des sexualités, même s'il n'a pas vocation à être, en tant que tel, un ouvrage scientifique stricto sensu ${ }^{1}$. Les auteures en effet retranscrivent avant tout les données de terrain et ne construisent pas d'analyse de ces récits. Leur but était, à partir des portraits qui ressortent de ces narrations, « de rendre compte des enchevêtrements de problématiques construites dans l'interaction, et de permettre au lecteur d'en tirer des questionnements et réflexions étoffés de l'expérience et du vécu» (p.7) des personnes qui se sont livrées. Notons dès le départ que le sous-titre donné à l'ouvrage qui entend parler des homosexualités en général peut paraitre trompeur, puisqu'il est essentiellement question d'homosexualités masculines.

\section{Déni, silences, violences et « accommodements » autour de l'homosexualité}

3 En 2012, les statistiques montrent une prévalence du VIH allant de 0.1 à 5.6\% parmi la population générale des différents contextes étudiés, et de 2.4 au Burundi à $37.2 \%$ au Cameroun parmi les HSH (Hommes ayant des relations Sexuelles avec des Hommes), l'un des "groupes cibles» des programmes de lutte contre le virus. Ces chiffres - qui ont certainement été actualisés depuis - rappellent, s'il en était besoin, l'importance et l'urgence de conduire des études tant sur l'épidémie que sur ceux qui la subissent ${ }^{2}$.

D'un point de vue juridique, l'homosexualité est pénalisée dans cinq pays sur les sept concernés par l'étude. Si au Burkina Faso et en Côte d'Ivoire il n'existe aucune mention dans la loi de l'homosexualité, le code pénal en vigueur au Cameroun, Burundi, Togo, en Algérie et au Maroc prévoit des amendes et des peines d'emprisonnement de six mois à trois ans pour toute personne ayant des rapports sexuels avec une autre personne de son sexe (p. 24-32). Cependant, la prévalence du virus dans les pays étudiés et l'intégration des HSH comme groupes cibles de la lutte contre le sida de façon internationale permettent une "bio-légitimité ${ }^{3}$ " de ceux-ci. Les auteures soulignent cependant qu'elle est avant tout « sur le papier » (p. 35), et que peu de moyens concrets sont mis en œuvre sur le terrain par les États étudiés. Outre la disparité des contextes, les différentes dispositions tant en matière de santé publique qu'en faveur des droits des minorités sexuelles sont bien souvent remises en cause, du fait qu'elles peuvent être perçues comme « une nouvelle forme d'impérialisme occidental, via l'exportation et l'imposition de modèles identitaires et sexuels considérés comme déviants et "non africains" »(p.36), complexifiant les enjeux géopolitiques et créant des situations paradoxales.

5 Dans certains de ces contextes, la loi répressive et la peur de la police et de ses violences contribuent à créer un climat oppressant pour les personnes homosexuelles : les idées liberticides, sexistes et homophobes sont alors véhiculées par les médias, les responsables politiques et religieux (montrant une instrumentalisation de la question), 
voire dans certains cas par les familles et les proches, du fait surtout d'une méconnaissance à l'égard de cette population. De ce fait, même dans des pays où l'homosexualité n'est pas pénalisée, les stigmatisations et discriminations à l'encontre des HSH sont présentes, comme le soulignent certains acteur.es interrogé.es pendant l'enquête. Cependant ces discriminations varient énormément selon les lieux et les périodes - les récits sont ainsi nuancés et loin d'être univoques. L'anonymisation nécessaire, tant des noms que de l'origine géographique, au vu des risques encourus par cette prise de parole, empêche par contre d'associer chaque récit au pays dont il est question.

6 Ces différents contextes politiques et sociaux justifient ainsi le fil conducteur choisi par les auteures une fois les récits récoltés et rassemblés : un " jeu d'ombres et de lumière » (p. 20), tant d'un point de vue collectif qu'individuel. Le titre même fait référence à un adage ouest-africain, rapporté par un médecin exerçant dans l'une des associations où a été menée l'enquête :

"Qu'on le veuille ou non, les hommes qui ont des relations sexuelles avec d'autres hommes existent. Il faut faire avec cette population. Comme on dit chez nous : "On ne cache pas le soleil avec la main". Tu as beau le cacher, le soleil est toujours là ! Ces hommes existent, on le sait. Il faut s'occuper d'eux, et c'est tout. » (p. 20)

7 La lutte pour la reconnaissance est au cœur même des préoccupations des acteur.es interrogé.es, qui font tous partie d'associations africaines partenaires d'AIDES et de Sidaction, ou qui bénéficient de financements d'autres structures luttant contre le sida. Leurs trajectoires militantes et personnelles sont retranscrites plus en détail dans les deux parties suivantes de l'ouvrage.

\section{Donner la voix aux principaux concerné.es}

8 La principale nouveauté du livre est ainsi de ne pas rapporter la parole de ceux qui se positionnent contre les projets à destination des $\mathrm{HSH}$, ce qui évite d'alimenter les discours alarmistes voire scandalisants à propos d'une "Afrique homophobe ", en mettant en avant celles et ceux qui travaillent au quotidien dans le développement d'actions à destination des personnes homosexuelles. La seconde partie de l'ouvrage, «Parcours d'émergence », est ainsi composée de trois récits de responsables associatifs dont les organisations se sont engagées dans ce domaine. Si l'épidémie émerge dans les années 1980, et si pendant près de vingt ans la priorité fut donnée à la question de l'accès aux soins des personnes vivant avec le VIH, l'on note un retour de la prévention depuis le milieu des années 2000. Les difficultés juridiques et politiques déjà mentionnées s'additionnent ici aux obstacles budgétaires, puisque les financements dont bénéficient les associations locales sont tributaires des fonds mondiaux et européens, du fait de la globalisation de la lutte contre le sida. Les adaptations locales sont diverses selon les contextes et les buts affichés des structures, devant constamment employer des tournures rhétoriques pour ne pas exposer leurs actions à l'égard des homosexuels. De fait, la promotion de la santé publique ou la lutte pour les droits humains sans distinction servent bien souvent de carapace et de stratégie afin de détourner le regard et brouiller les pistes, permettant une meilleure prise en charge des HSH et la création d'espaces sécurisants à leur attention. Cela permet également de souligner la perméabilité entre les groupes considérés comme particulièrement 
vulnérables à l'épidémie, puisque des activités à destination des «travailleurs et travailleuses du sexe» sont également développées, et qu'on ne peut toucher l'homosexualité sans mentionner la bisexualité - le fait de se marier constituant bien souvent une couverture pour les hommes aimant d'autres hommes. Le vécu de la séropositivité est comparé au vécu de l'homosexualité par l'une des responsables associatives (p.52), mettant en avant l'auto-stigmatisation qui touche les personnes concernées, alors qu'un autre rappelle les pressions et violences à leur égard (dénonciations, lynchages, prison, outing instrumentalisé à des fins politiques, etc.), et ainsi la fragilité combinée des individus et des structures dans ces espaces. La peur ne touche pas simplement ceux dont la sexualité est niée et décriée, mais également celles et ceux qui luttent au quotidien contre cette situation, rappelant les risques d'une prise de parole publique sur le sujet, pouvant «exposer tout le monde» (p. 64): autres militant.es, familles, proches ; et ainsi mettre à mal les collectifs.

9 À ces premiers entretiens succèdent quatre autres récits dans une troisième partie, "Travailler auprès de ses pairs ", qui constitue les pages les plus intéressantes de l'ouvrage. Les auteures franchissent un pas de plus en retranscrivant une « histoire par le bas » à la fois des vécus de l'homosexualité et de la prévention, donnant cette fois-ci la voix aux pairs-éducateurs qui sillonnent les villes à l'extérieur des murs associatifs à la rencontre d'autres $\mathrm{HSH}$, et qui racontent leurs vécus professionnels, mais également personnels et intimes. Lucides, parfois émouvantes, voire terribles, leurs histoires sont narrées non sans humour et permettent d'approcher au plus près leurs réalités quotidiennes. Les personnes ayant accepté de se livrer naviguent entre plusieurs sphères sociales et font constamment évoluer leurs identités au gré de leurs itinéraires. Là encore, les tactiques et stratégies déployées pour vivre en secret constituent une bonne part de leurs vies ordinaires. Mais les parcours retranscrits font aussi la part belle aux sociabilités et solidarités multiples, aux désirs, aux rêves, à l'invention d'espaces où vivre son homosexualité, et ainsi l'agency des personnes émerge, passant de leur statut supposément victimaire en acteurs tant des luttes que des vies menées.

\section{Compositions et recompositions des genres}

10 Finalement, outre le fait qu'elle permette au travers des associations de créer peu à peu des emplois, bien que précaires, destinés aux HSH, l'épidémie devient le prétexte pour prendre la parole, pour se rencontrer, se rassembler et (se) raconter. Elle devient un lieu d'observation des sexualités et, de fait, émerge une multiplicité de subjectivités qui interrogent le genre. Les définitions mouvantes des identités sont rappelées par les auteures en préambule de cette seconde moisson de récits, qui soulignent la pluralité des noms et locutions employés pour parler de l'homosexualité, composant un vocabulaire empruntant tant aux langues des pays dans lesquels l'enquête a été réalisée qu'aux terminologies occidentales qui ne cessent d'influencer, à différents degrés, les formes langagières. Là encore, les stratégies de dissimulation se lisent dans la langue, qui devient performative. Si Amin souligne à la fois les tensions et rivalités entre les " discrets » et les « efféminés » (p. 90-91), rappelant de ce fait la difficulté à rattacher les identités homosexuelles diverses à une catégorie importée des États-Unis et créée par les ONG et les pouvoirs publics (HSH), cet acronyme est pourtant beaucoup utilisé et réapproprié, explique Paul, "comme un code» (p.116), n'étant connu que des personnes gravitant autour des associations ${ }^{4}$. Cette ruse de chiffrage des mots est 
commune et déclinée à un niveau infra : « Pour notre sécurité, quand on veut parler des trans, des passifs, des actifs et des autres, on utilise des mots différents » (p. 101). Dans un autre domaine de la vie sociale, le fait de parler « en paraboles » avec ses proches permet à la fois de tester son interlocuteur et de ne pas se dévoiler complètement, dans le cas où l'identité si longtemps cachée ne serait pas pleinement acceptée (p. 124).

11 Communiquer en inversant le genre dans d'autres situations peut également constituer un moyen d'aborder de façon détournée l'homosexualité, alors que l'auto-définition évolue en permanence - Ariane ayant réalisé l'entretien au masculin, et décidé, à sa relecture, de parler d'elle au féminin. De là apparaît le principal manque que l'on peut ressentir à la lecture de l'ouvrage : l'occultation de l'homosexualité féminine. Si les contextes oppressifs dans lesquels évoluent les personnes expliquent en partie cela, de même que le sida, qui constitue la porte d'entrée sur l'homosexualité, tend à passer sous silence cette réalité (la transmission du VIH entre femmes étant moindre, elles ne sont pas considérées comme un "groupe à risque »), il est gênant qu'à l'exception d'une note, aucune autre mention n'en soit faite. Cela laisse finalement la porte ouverte à de nouveaux terrains d'étude.

12 Il est par ailleurs fort probable que le peu de temps passé dans chaque pays (environ une semaine) ait restreint l'accès à cette autre réalité. Autre limite: la voix des personnes homosexuelles éloignées des associations de lutte contre le sida est également absente. L'ouvrage n'en constitue pas moins un point de départ pouvant servir à lancer des recherches plus poussées sur ces questions, et à faire un nouveau bilan, sept années après cette enquête multi-située. Soulignons sur ce point l'audace des auteures d'avoir su proposer, en finalement peu de pages tant chaque pays mériterait une étude à part entière, un livre présentant une vision d'ensemble des expériences militantes et homosexuelles en Afrique francophone sans tomber dans une quelconque simplification, ni uniformisation, puisque la disparité et la complexité des situations locales et des points de vue sont sans cesse rappelées, ce qui, on s'en doute, ne constitue pas l'une des moindres difficultés d'écriture.

\section{Hybridité de l'ouvrage et multiplicité des récits}

C'est enfin cette démarche rédactionnelle particulière qu'il convient de saluer : alors que d'ordinaire les études commanditées par les associations de lutte contre le sida, et plus généralement de santé publique, se doivent de rester dans des formes très codifiées, Lucille Gallardo et Laura Spica ont ici proposé un ouvrage tiré de l'étude qui sort complètement de ces cadres de restitution normés 5 . La place de la sérigraphie est centrale et non simplement illustrative, constituant un langage en soi et permettant de faire de l'ouvrage un outil pour de multiples publics sortant des rangs stricts de la recherche-action ou de l'académisme - on pense notamment aux professeurs du secondaire et aux lycéens, qui peuvent aussi s'en emparer. En cela, La main devant le soleil est précurseur, à un moment où l'on note l'explosion de la réflexion autour de nouveaux types d'écriture des sciences humaines et sociales depuis quelques années. Pareillement, la mise en place graphique d'un système de méta-texte tout au long de l'ouvrage (extraits d'entretiens, notes en marge faisant référence directe aux cas étudiés, exergues, mots soulignés) permet une lecture multiple de celui-ci, cassant les codes ordinaires de la narration. En ressort un ouvrage hybride, portant de multiples récits et de multiples voix, des partenaires institutionnels aux individus auxquels sont 
destinés les programmes de lutte contre le sida, les chercheuses tendant même à s'effacer - peut-être même trop - derrière cette pluralité de portraits et cet " assemblage artisanal d'histoires vécues, racontées, entendues, écrites et illustrées par autant de voix et de mains » (p. 7).

Si le but des auteures était, simplement et humblement, de donner à entendre et à lire ces hommes et ces femmes, la richesse de l'ouvrage et des problématiques qui en découlent montre que ce dessein a largement été dépassé.

\section{BIBLIOGRAPHIE}

GOUYON, Marien \& MUSSO, Sandrine. 2017. « Luttes contre le sida et luttes LGBT au Maroc. Notes exploratoires sur les enjeux d'une imbrication » L'Année du Maghreb 17 : 201-217.

FASSIN, Éric. 2005. « Le sens de la santé. Anthropologie des politiques de la vie » in Anthropologie médicale. Ancrages locaux, défis globaux, SAILLANT, Francine \& GENEST, Serge. Québec : Les Presses de l'Université Laval/Paris : Anthropos, 383-399.

SERVY, Alice. 2017. «AIDS IS HERE !». Prévenir les infections sexuellement transmissibles à Port-Vila, Vanuatu. Thèse d'anthropologie sociale et ethnologie. Marseille : EHESS.

SPICA, Laura. 2012. Le pied sur la braise. Visages et rivages du handicap en Méditerranée. Marseille : Accatone.

THEVENET, Charlotte. 2017. Recension de « Calogero Giametta. 2017. The Sexual Politics of Asylum. Sexual Orientations and Gender Identity in the UK Asylum System et Thibaut Raboin. 2017. Discourses on LGBT Asylum in the UK. Constructing a queer haven », GLAD! [En ligne], 03 | 2017, https://www.revue-glad.org/783

\section{NOTES}

1. Édité par deux associations (Le Tamis et AIDES), dont une qui intervient dans les programmes de lutte contre le sida en Afrique, ce recueil est également un outil de sensibilisation à la question traitée. Même si à la lecture de l'ouvrage AIDES apparaît finalement peu, son existence s'inscrit dans le cadre d'une collaboration où l'association souhaitait valoriser son action et celle de ses partenaires : c'est dans ce contexte que les entretiens ont été produits à l'origine. Les auteures ont conscience du biais potentiel dans l'exposition des résultats et annoncent dès le prologue qu'il ne s'agit «ni d'un livre scientifique, ni d'un écrit littéraire » (p. 7) mais plutôt du résultat d'un "bricolage" né de ces différentes façons de faire de l'anthropologie, du fait des financements divers - et souvent absents - du monde de la recherche actuel. Cependant, du fait de la formation et de la pratique professionnelle des auteures (le Tamis est une association de sciences sociales), des références scientifiques émaillent l'ouvrage, préfacé par Patrick Awondo (anthropologue spécialiste de ces questions au Cameroun).

2. Pour un bilan plus récent sur la question au Maroc, et notamment sur les enjeux de la convergence des luttes pour les droits des LGBT et contre le sida, voir Gouyon, Marien \& Musso, 
Sandrine. 2017. « Luttes contre le sida et luttes LGBT au Maroc. Notes exploratoires sur les enjeux d'une imbrication » L'Année du Maghreb 17 : 201-217.

3. Cette notion apparaît dans différents travaux d'Éric Fassin, qui, en partant de la notion de biopouvoir foucaldienne, considère, plutôt qu'un « pouvoir sur la vie » un « pouvoir de la vie », et donc une attention et une reconnaissance portées par la société aux corps souffrants et malades (voir par exemple sa contribution de 2005, «Le sens de la santé. Anthropologie des politiques de la vie » in Anthropologie médicale. Ancrages locaux, défis globaux, Saillant, Francine \& Genest, Serge. Québec : Les Presses de l'Université Laval/Paris : Anthropos, 383-399).

4. Du fait du cadre dans lequel le livre a été produit, cette catégorie n'est pas mise directement en question par les auteures. Pour aller plus loin concernant ce point, voir la partie de la thèse d'Alice Servy consacrée à ce sujet dans le contexte du Vanuatu (Servy, Alice. 2017. "AIDS IS HERE ! ». Prévenir les infections sexuellement transmissibles à Port-Vila, Vanuatu. Thèse d'anthropologie sociale et ethnologie. Marseille: EHESS). La question plus large de l'inadéquation souvent présente entre les catégories créées par les institutions et le vécu et les trajectoires des personnes concernées en matière de genre et de sexualité est explicitée dans la double recension des travaux de Calogero Giametta et Thibaut Raboin, qui concernent le droit d'asile et les personnes LGBT, dans le troisième numéro de Glad! (Charlotte Thevenet, "Calogero Giametta. 2017. The Sexual Politics of Asylum. Sexual Orientations and Gender Identity in the UK Asylum System et Thibaut Raboin. 2017. Discourses on LGBT Asylum in the UK. Constructing a queer haven ", GLAD! [En ligne], 03 | 2017, mis en ligne le 10 décembre 2017, consulté le 15 novembre 2018. URL : https:// www.revue-glad.org/783)

5. Un ouvrage similaire est paru deux ans plus tôt, l'année où l'association qui a co-édité le livre est née (Spica, Laura. 2012. Le pied sur la braise. Visages et rivages du handicap en Méditerranée. Marseille : Accatone).

\section{INDEX}

Thèmes : Actualités

Mots-clés : homosexualités masculines, sida, récits, associations, militants

Keywords : male homosexuality, AIDS, narratives, associations, activists

\section{AUTEURS}

\section{FLEUR BEAUVIEUX}

Fleur Beauvieux est docteure en histoire rattachée au centre Norbert Elias. Elle a travaillé sur la dernière épidémie de peste marseillaise du XVIII ${ }^{\mathrm{e}}$ siècle, en interrogeant notamment la façon dont les habitants et habitantes continuaient de vivre malgré la violence du contexte épidémique et le risque de contagion. 